\title{
Power Control for Fading Cooperative Multiple Access Channels
}

\author{
Onur Kaya, Member, IEEE, and Sennur Ulukus, Member, IEEE
}

\begin{abstract}
For a fading Gaussian multiple access channel with user cooperation, we obtain the power allocation policies that maximize the average rates achievable by block Markov superposition coding, subject to average power constraints. The optimal policies result in a coding scheme that is simpler than the one for a general multiple access channel with generalized feedback. This simpler coding scheme also leads to the possibility of formulating an otherwise non-concave optimization problem as a concave one. Using the perfect channel state information available at the transmitters to adapt the powers, we demonstrate gains over the achievable rates for existing cooperative systems.
\end{abstract}

Index Terms - Cooperative diversity, fading, multiple access channel, power control, user cooperation.

\section{INTRODUCTION}

$\mathbf{I}$ NCREASING demand for higher rates in wireless communication systems has recently triggered major research efforts to characterize the capacities of such systems. The wireless medium brings along its unique challenges such as fading and multiuser interference, which make the analysis of communication systems more complicated. On the other hand, the same challenging properties of such systems are what give rise to the concepts such as diversity, over-heard information, etc., which can be carefully exploited to the advantage of the network capacity.

In the early 1980s, several problems which form a basis for the idea of user cooperation in wireless networks were solved. First, the case of a two user multiple access channel (MAC) where both users have access to the channel output was considered by Cover and Leung [1], and an achievable rate region was obtained for this channel. Willems and van der Meulen then demonstrated [2] that the same rate region is achievable if there is a feedback link to only one of the tansmitters from the channel output.

The capacity region of the MAC with partially cooperating encoders was obtained by Willems in [3]. In this setting, the encoders are assumed to be connected by finite capacity communication links, which allow the cooperation. Willems

Manuscript received November 11, 2005; revised July 7, 2006 and April 24, 2007; accepted April 28, 2007. The editor coordinating the review of this paper and approving it for publication was A. Yener. This work was supported by NSF Grants ANI 02-05330, CCR 03-11311 and CCF 04-47613, ARL/CTA Grant DAAD 19-01-2-0011, and the Scientific \& Technological Research Council of Turkey Grant 106E018; and was presented in part at the IEEE International Conference on Wireless Networks, Communications, and Mobile Computing, Maui, HI, June 2005.

O. Kaya is with the Department of Electronics Engineering, Işı University, Istanbul, Turkey (email: onurkaya@ isikun.edu.tr).

S. Ulukus is with the department of Electrical and Computer Engineering, University of Maryland, College Park, MD, USA (email: ulukus@eng.umd.edu).

Digital Object Identifier 10.1109/TWC.2007.05858 and van der Meulen also considered a limiting case of cooperation where the encoders "crib" from each other, that is, they learn each others' codewords before the next transmission [4]. Several scenarios regarding which encoder(s) crib, and how much of the codewords the encoders learn, are treated and the capacity region for each case is obtained in [4]. The capacity of such channels are an upper bound to the rates achievable by cooperative schemes, since in the case of cribbing encoders, the sharing of information comes for free, i.e., the transmitters do not allocate any resources such as powers, to establish common information.

For a MAC with generalized feedback, an achievable rate region was obtained in [5] by Carleial. A simpler and larger rate region for the same channel was then obtained by Willems, van der Meulen and Schalkwijk in [6]. This channel model is worth special attention as far as the wireless channels are concerned, since it models the over-heard information by the transmitters. In particular, for a two user MAC with generalized feedback described by $\left(\mathcal{X}_{1} \times\right.$ $\left.\mathcal{X}_{2}, P\left(y, y_{1}, y_{2} \mid x_{1}, x_{2}\right), \mathcal{Y} \times \mathcal{Y}_{1} \times \mathcal{Y}_{2}\right)$, where user 1 has access to channel output $Y_{1}$ and user two has access to channel output $Y_{2}$, an achievable rate region is obtained by using a superposition block Markov encoding scheme, together with backward decoding, where the receiver waits to receive all $B$ blocks of codewords before decoding.

Recently, Sendonaris, Erkip and Aazhang have successfully employed the results of these rather general problems, particularly that of generalized feedback, to a Gaussian MAC in the presence of fading, leading to user cooperation diversity and higher rates [7]. In this setting, both the receiver and the transmitters receive noisy versions of the transmitted messages, and slightly modifying the basic relay channel case, the transmitters form their codewords not only based on their own information, but also on the information they have received from each other. It is assumed in [7] that channel state information for each link is known to the corresponding receiver on that link, and also phase of the channel state needs to be known at the transmitters in order to obtain a coherent combining gain. The achievable rate region is shown to improve significantly over the capacity region of the MAC with non-cooperating transmitters, especially when the channel between the two users is relatively good on average.

There has also been some recent work on user cooperation systems under various assumptions on the available channel state information, and the level of cooperation among the users. Laneman, Tse and Wornell [8] have characterized the outage probability behavior for a system where the users are allowed to cooperate only in half-duplex mode, and where no channel state information is available at the transmitters. 
For the relay channel, which is a special one-sided case of user cooperation, Host-Madsen and Zhang [9] have solved for power allocation policies that optimize some upper and lower bounds on the ergodic capacity when perfect channel state information is available at the transmitters and the receiver. For a user cooperation system with finite capacity cooperation links, Erkip [10] has proposed a suboptimal solution to the problem of maximizing the sum rate in the presence of full channel state information, where it was also noted that the resulting optimization problem is non-convex.

In this paper, we consider a two user fading cooperative Gaussian MAC with complete channel state information at the transmitters and the receiver, and average power constraints on the transmit powers. Note that, this requires only a small quantity of additional feedback, namely the amplitude information on the forward links, over the systems requiring coherent combining [7]. In this case, the transmitters can adapt their coding strategies as a function of the channel states, by adjusting their transmit powers [11]-[13]. We characterize the optimal power allocation policies which maximize the set of ergodic rates achievable by block Markov superposition coding. To this end, we first prove that the seemingly nonconcave optimization problem of maximizing the achievable rates can be reduced to a concave problem, by noting that some of the transmit power levels are essentially zero at every channel state, which also reduces the dimensionality of the problem. By this, we also show that the block Markov superposition coding strategy proposed in [6] and employed in [7] for a Gaussian channel can be simplified considerably by making use of the channel state information. Due to the nondifferentiable nature of the objective function, we first replace the optimization problem with an equivalent differentiable one, and find the structure of the optimal power allocation policies by using convex optimization techniques. Then, we use sub-gradient methods to numerically obtain the optimal power distributions that maximize the achievable rates, and we provide the corresponding achievable rate regions for various fading distributions. We demonstrate that controlling the transmit powers in conjunction with user cooperation provides significant gains over the existing rate regions for cooperative systems.

\section{System Model}

We consider a two user fading Gaussian MAC, where both the receiver and the transmitters receive noisy versions of the transmitted messages, as illustrated in Figure 1. The system is modelled by,

$$
\begin{aligned}
& Y_{0}=\sqrt{h_{10}} X_{1}+\sqrt{h_{20}} X_{2}+Z_{0} \\
& Y_{1}=\sqrt{h_{21}} X_{2}+Z_{1} \\
& Y_{2}=\sqrt{h_{12}} X_{1}+Z_{2}
\end{aligned}
$$

where $X_{i}$ is the symbol transmitted by node $i, Y_{i}$ is the symbol received at node $i$, and the receiver is denoted by $i=0 ; Z_{i}$ is the zero-mean additive white Gaussian noise at node $i$, having variance $\sigma_{i}^{2}$, and $\sqrt{h_{i j}}$ are the random fading coefficients, the instantaneous realizations of which are assumed to be known by both the transmitters and the

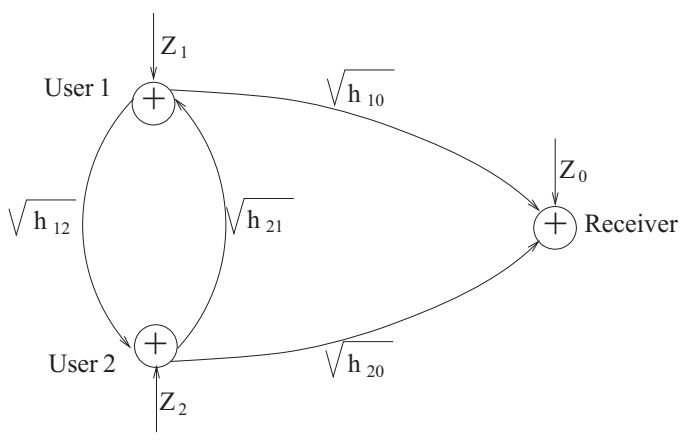

Fig. 1. Two user fading cooperative MAC.

receiver. We assume that the channel variation is slow enough so that the fading parameters can be tracked accurately at the transmitters, yet fast enough to ensure that the long term ergodic properties of the channel are observed within the blocks of transmission [14]. The transmitters are capable of making decoding decisions based on the signals they receive and thus can form their transmitted codewords not only based on their own information, but also based on the information they have received from each other. This channel model is a special case of the MAC with generalized feedback [6]. The achievable rate region is obtained by using a superposition block Markov encoding scheme, together with backwards decoding, where the receiver waits to receive all $B$ blocks of codewords before decoding. Note that, an alternative is to use window decoding, which starts the decoding process only after a delay of one block. However, although window decoding has recently been shown to achieve the same rates as backwards decoding under several relay channel models [15], [16], as well as the same sum rate as backwards decoding in the fully cooperative MAC scenario [17], it is in general inferior to backwards decoding in terms of the entire achievable rate region when block Markov encoding is used [18]. Therefore, in this paper we stick with backwards decoding.

For the Gaussian case, the superposition block Markov encoding is realized as follows [7]: the transmitters allocate some of their powers to establish some common information in every block, and in the next block, they coherently combine part of their transmitted codewords. In the presence of channel state information, by suitably modifying the coding scheme given by [7] to accommodate for channel adaptive coding strategies, the encoding is performed by

$$
X_{i}=\sqrt{p_{i 0}(\mathbf{h})} X_{i 0}+\sqrt{p_{i j}(\mathbf{h})} X_{i j}+\sqrt{p_{U_{i}}(\mathbf{h})} U
$$

for $i, j \in\{1,2\}, i \neq j$, where $X_{i 0}$ carries the fresh information intended for the receiver, $X_{i j}$ carries the information intended for transmitter $j$ for cooperation in the next block, and $U$ is the common information sent by both transmitters for the resolution of the remaining uncertainty from the previous block, all chosen from unit-power Gaussian distributions. All the transmit power is therefore captured by the power levels associated with each component, i.e., $p_{i 0}(\mathbf{h})$, $p_{i j}(\mathbf{h})$ and $p_{U_{i}}(\mathbf{h})$, which are required to satisfy the average power constraints,

$$
E\left[p_{i 0}(\mathbf{h})+p_{i j}(\mathbf{h})+p_{U_{i}}(\mathbf{h})\right]=E\left[p_{i}(\mathbf{h})\right] \leq \bar{p}_{i}, \quad i=1,2 .
$$




$$
\begin{aligned}
R_{1}<E\left[\log \left(1+\frac{h_{12} p_{12}(\mathbf{h})}{h_{12} p_{10}(\mathbf{h})+\sigma_{2}^{2}}\right)+\log \left(1+\frac{h_{10} p_{10}(\mathbf{h})}{\sigma_{0}^{2}}\right)\right] \\
R_{2}<E\left[\log \left(1+\frac{h_{21} p_{21}(\mathbf{h})}{h_{21} p_{20}(\mathbf{h})+\sigma_{1}^{2}}\right)+\log \left(1+\frac{h_{20} p_{20}(\mathbf{h})}{\sigma_{0}^{2}}\right)\right] \\
R_{1}+R_{2}<\min \left\{E\left[\log \left(1+\frac{h_{10} p_{1}(\mathbf{h})+h_{20} p_{2}(\mathbf{h})+2 \sqrt{h_{10} h_{20} p_{U_{1}}(\mathbf{h}) p_{U_{2}}(\mathbf{h})}}{\sigma_{0}^{2}}\right)\right],\right. \\
\left.E\left[\log \left(1+\frac{h_{10} p_{10}(\mathbf{h})+h_{20} p_{20}(\mathbf{h})}{\sigma_{0}^{2}}\right)+\log \left(1+\frac{h_{12} p_{12}(\mathbf{h})}{h_{12} p_{10}(\mathbf{h})+\sigma_{2}^{2}}\right)+\log \left(1+\frac{h_{21} p_{21}(\mathbf{h})}{h_{21} p_{20}(\mathbf{h})+\sigma_{1}^{2}}\right)\right]\right\}
\end{aligned}
$$

Following the results in [7], it can be shown that the achievable rate region $\mathcal{R}$ is given by the convex hull of all rate pairs satisfying (6)-(8) at the top of the this page, where the convex hull is taken over all power allocation policies that satisfy (5).

For a given valid power allocation $\mathbf{p}(\mathbf{h})$, let us denote the rate region in (6)-(8) by $\mathcal{R}(\mathbf{p}(\mathbf{h}))$. This region is either a pentagon or a triangle, since, unlike the traditional MAC, the sum rate constraint in (8) may dominate the individual rate constraints completely. Then, the overall achievable rate region $\mathcal{R}$ may alternatively be represented as the convex hull of the union of all such regions, i.e.,

$$
\mathcal{R}=\operatorname{conv}\left(\bigcup_{\left\{E\left[p_{i}(\mathbf{h})\right] \leq \bar{p}_{i}, i=1,2\right\}} \mathcal{R}(\mathbf{p}(\mathbf{h}))\right)
$$

Our goal is to find the power allocation policies that maximize the rate tuples on the rate region boundary.

\section{Structure of the Sum Rate AND the OPTIMAL POLICIES}

We first consider the problem of optimizing the sum rate of the system, as it will also shed some light onto the optimization of an arbitrary point on the rate region boundary. The sum rate (8) is not a concave function of the vector of variables $\mathbf{p}(\mathbf{h})=\left[p_{10}(\mathbf{h}) p_{12}(\mathbf{h}) p_{U_{1}}(\mathbf{h}) p_{20}(\mathbf{h})\right.$

$\left.p_{21}(\mathbf{h}) p_{U_{2}}(\mathbf{h})\right]$, due to the existence of variables in the denominators. In what follows, we show that for the sum rate to be maximized, for every given $\mathbf{h}$, at least two of the four components of $\left[p_{10}(\mathbf{h}) p_{12}(\mathbf{h}) p_{20}(\mathbf{h}) p_{21}(\mathbf{h})\right]$ should be equal to zero, which reduces the dimensionality of the problem and yields a concave optimization problem.

Proposition 1: Let the effective channel gains normalized by the noise powers be defined as $s_{i j}=h_{i j} / \sigma_{j}^{2}$. Then, for the power control policy $\mathbf{p}^{*}(\mathbf{h})$ that maximizes $(8)$, we need

1) $p_{10}^{*}(\mathbf{h})=p_{20}^{*}(\mathbf{h})=0$, if $s_{12}>s_{10}$ and $s_{21}>s_{20}$

2) $p_{10}^{*}(\mathbf{h})=p_{21}^{*}(\mathbf{h})=0$, if $s_{12}>s_{10}$ and $s_{21} \leq s_{20}$

3) $p_{12}^{*}(\mathbf{h})=p_{20}^{*}(\mathbf{h})=0$, if $s_{12} \leq s_{10}$ and $s_{21}>s_{20}$

4) $p_{12}^{*}(\mathbf{h})=p_{21}^{*}(\mathbf{h})=0$

$$
\left.\begin{array}{c}
\text { OR } \\
p_{10}^{*}(\mathbf{h})=p_{21}^{*}(\mathbf{h})=0 \\
p_{12}^{*}(\mathbf{h})=p_{20}^{*}(\mathbf{h})=0
\end{array}\right\} \text { if } s_{12} \leq s_{10} \text { and } s_{21} \leq s_{20}
$$

Proof: To simplify the notation, let us drop the dependence of the powers on the channel states, whenever such dependence is obvious from the context. Let $p_{i}=p_{i 0}+p_{i j}+p_{U_{i}}$ be the total power allocated to a given channel state. Let us define

$$
\begin{aligned}
& A=1+s_{10} p_{1}+s_{20} p_{2}+2 \sqrt{s_{10} s_{20} p_{U_{1}} p_{U_{2}}} \\
& B=\frac{1+s_{10} p_{10}+s_{20} p_{20}}{\left(1+s_{12} p_{10}\right)\left(1+s_{21} p_{20}\right)} \\
& C=\left(1+s_{12}\left(p_{10}+p_{12}\right)\right)\left(1+s_{21}\left(p_{20}+p_{21}\right)\right)
\end{aligned}
$$

Then, an equivalent representation of the sum rate in (8) is

$$
R_{\text {sum }}=\min \{E[\log (A)], E[\log (B C)]\}
$$

Now, let us arbitrarily fix the total power level, $p_{i}$, as well as the power level used for cooperation signals, $p_{U_{i}} \leq$ $p_{i}$, allocated to a given state for each user. For each such allocation, the quantities $A$ and $C$ appearing in the sum rate expression are fixed, i.e., allocating the remaining available power $p_{i}-p_{U_{i}}$ among $p_{i 0}$ and $p_{i j}$ will not alter these quantities. Note that, such allocation also does not alter the total power consumption at the given state, so we may limit our attention to the maximization,

$$
\begin{aligned}
\max _{\left\{p_{10}, p_{20}\right\}} & B\left(p_{10}, p_{20}\right) \\
\text { s.t. } & p_{10}+p_{12}=p_{1}-p_{U_{1}} \\
& p_{20}+p_{21}=p_{2}-p_{U_{2}}
\end{aligned}
$$

The partial derivatives of $B$ with respect to $p_{10}$ and $p_{20}$ are

$$
\begin{aligned}
\frac{\partial B}{\partial p_{10}} & =\frac{s_{10}-s_{12}\left(1+s_{20} p_{20}\right)}{\left(1+s_{12} p_{10}\right)^{2}\left(1+s_{21} p_{20}\right)} \\
\frac{\partial B}{\partial p_{20}} & =\frac{s_{20}-s_{21}\left(1+s_{10} p_{10}\right)}{\left(1+s_{21} p_{20}\right)^{2}\left(1+s_{12} p_{10}\right)}
\end{aligned}
$$

Therefore, we make the following conclusions:

1) $s_{12}>s_{10}, s_{21}>s_{20}$. Then, $\frac{\partial B}{\partial p_{10}}<0$ and $\frac{\partial B}{\partial p_{20}}<0$, i.e., $B\left(p_{10}, p_{20}\right)$ is monotonically decreasing in both $p_{10}$ and $p_{20}$, therefore the sum rate is maximized at $p_{10}=$ $p_{20}=0$.

2) $s_{12}>s_{10}, s_{21} \leq s_{20}$. Then, $\frac{\partial B}{\partial p_{10}}<0$, and the function is maximized at $p_{10}=0$ for any $p_{20}$. But this gives $\left.\frac{\partial B}{\partial p_{20}}\right|_{p_{10}=0}>0$, meaning $p_{20}$ should take its maximum possible value, i.e., $p_{21}=0$.

3) $s_{12} \leq s_{10}, s_{21}>s_{20}$. Follows the same lines of case 2 with roles of users 1 and 2 reversed.

4) $s_{12} \leq s_{10}, s_{21} \leq s_{20}$. In this case, the partial derivatives of $B$ can be both made equal to zero within the constraint set, yielding a critical point. However, using second order derivative tests, it is possible to show that the determinant of the Hessian matrix is negative, and 
therefore this solution corresponds to a saddle point, and $B$ is again maximized at one of the boundaries, $p_{10}=0$, $p_{20}=0, p_{10}=p_{1}-p_{U_{1}}, p_{20}=p_{2}-p_{U_{2}}$, i.e., two of the components of the optimal power vector are again guaranteed to be zero. Inspection of the gradient on these boundary points yields one of the three corner points $\left\{\left(p_{1}-p_{U_{1}}, 0\right),\left(0, p_{2}-p_{U_{2}}\right),\left(p_{1}-p_{U_{1}}, p_{2}-p_{U_{2}}\right)\right\}$ as candidates for optimal solution in case 4 .

Although two of the components of the power vector in case 4 are guaranteed to be equal to zero, which ones will be zero depends on the $p_{i}$ and $p_{U_{i}}$ that we fixed, therefore we are not able to completely specify the solution, independent of $p_{i}$ and $p_{U_{i}}$, in this case. On the other hand, the settings of interest to us are those where the channels between the cooperating users are on average much better than their direct links, since it is in these settings when cooperative diversity yields high capacity gains [7]. In such scenarios, the probability of both users direct link gains exceeding their corresponding cooperation link gains (case 4) is very low. Therefore, which of the three possible operating points is chosen is not of practical importance, and we can safely fix the power allocation policy to one of them to carry on with our optimization problem for other variables. Although admittedly this argument is likely to cause some suboptimality in our scheme, as will be seen in the numerical examples, we still obtain a significant gain in the achievable rates.

The significance of Proposition 1 is two-fold. Firstly, given a channel state, it greatly simplifies the well known block Markov coding, in a very intuitive way: if the direct links of both users are inferior to their cooperation links, the users do not transmit direct messages to the receiver as a part of their codewords, and they use each other as relays. If one of the users' direct channel is better than its cooperation channel, and the other user is in the opposite situation, then the user with the strong direct channel chooses to transmit directly to the receiver, while the weaker direct channel user still chooses to relay its information over its partner. Second important implication of this result is that it now makes the problem of solving for the optimal power allocation policy more tractable, since it simplifies the constraint set on the variables, and more importantly, since this makes the sum rate a concave function over the reduced set of constraints and variables.

Corollary 1: The sum rate $R_{\text {sum }}$ given by (8) is a concave function of $\mathbf{p}(\mathbf{h})$, over the reduced constraint set described by Proposition 1.

Proof: The proof of this result follows from directly substituting the zero power components into the sum rate expression in (13). Note that in each of the four cases, the second function in the minimization, i.e., $\log (B C)$ takes either the form $\log (1+a)+\log (1+b)$, or $\log (1+a+b)$, both of which are clearly jointly concave in $a$ and $b$. Also, $\log (A)$ is clearly a concave function of $\mathbf{p}(\mathbf{h})$ since it is a composition of a concave function with the concave and increasing logarithm. The desired result is obtained by noting that the minimum of two concave functions is concave.

Thus far we have discussed the structure of the sum rate, as well as some properties of the optimal power allocation that maximizes that rate. We now turn back to the problem of maximizing other rate points on the rate region boundary. To this end, we point out another remarkable property of the solution in Proposition 1. Consider maximizing the bound on $R_{1}$ in (6). For fixed $p_{U_{1}}$ and $p_{1}$, it is easy to verify that all of the available power should be allocated to the channel with the higher gain, i.e., if $s_{12}>s_{10}$, then we need $p_{10}=0$ and $p_{12}=p_{1}-p_{U_{1}}$. The same result also applies to $R_{2}$. But this shows that, the policies described in Proposition 1 completely agree with optimal policies for maximizing the individual rate constraints in cases 1-3, and they also agree if we choose the operating point in case 4 to be $p_{12}=0, p_{21}=0$. Therefore, the allocation in Proposition 1 enlarges the entire rate region in all directions (except for the subtlety in case 4 for the sum rate). Let us define $\Gamma$ as the set of valid power control policies $\mathbf{p}(\mathbf{h})$ which satisfy Proposition 1 , with $p_{12}=0, p_{21}=0$ being chosen in case 4 , i.e.,

$$
\begin{gathered}
\Gamma=\left\{\mathbf{p}(\mathbf{h}):\left\{E\left[p_{i}(\mathbf{h})\right], \leq \bar{p}_{i}\right\} \cap\left\{p_{i j}=0 \text { if } s_{i k}>s_{i j},\right\}\right. \\
i=1,2, j \neq k\}
\end{gathered}
$$

Then, the achievable rate region $\mathcal{R}$ given by (9) is equivalent to the following set of rates

$$
\mathcal{R}_{\Gamma}=\operatorname{conv}\left(\bigcup_{\mathbf{p}(\mathbf{h}) \in \Gamma} \mathcal{R}(\mathbf{p}(\mathbf{h}))\right)
$$

up to the possible minor sub-optimality that may be caused by fixing $p_{12}=0, p_{21}=0$ in case 4 . Thus, from now on we focus on the achievable rates given in (18).

Now, in order to obtain the power control policies that achieve arbitrary points on the rate region boundary, we first prove the following result stating that the union of achievable rate regions, over all valid power allocation policies satisfying the conditions given by Proposition 1, produces a convex region, and the convex hull operation in (18) is not needed and can be removed.

Lemma 1: The achievable rate region $\mathcal{R}_{\Gamma}$ defined in (18) is equivalent to

$$
\mathcal{R}_{\Gamma}=\bigcup_{\mathbf{p}(\mathbf{h}) \in \Gamma} \mathcal{R}(\mathbf{p}(\mathbf{h}))
$$

Proof: The proof of this lemma closely follows the proof of a similar result by Hanly and Tse [13], for the capacity region of traditional MAC. First, consider any two valid power allocation policies $\mathbf{p}^{1}(\mathbf{h}), \mathbf{p}^{2}(\mathbf{h})$ from the set $\Gamma$; and their respective sets of achievable rates $\mathcal{R}\left(\mathbf{p}^{1}(\mathbf{h})\right), \mathcal{R}\left(\mathbf{p}^{2}(\mathbf{h})\right)$. Let us define a new power control policy $\alpha \mathbf{p}^{1}(\mathbf{h})+(1-\alpha) \mathbf{p}^{2}(\mathbf{h})$, for $0 \leq \alpha \leq 1$, which also clearly satisfies the average power constraints and the conditions of Proposition 1. Since the power allocation policies satisfy the conditions in Proposition 1 by assumption, the upper bounds in (6)-(8) are concave. Then, using (6)-(8),

$$
\alpha \mathcal{R}\left(\mathbf{p}^{1}(\mathbf{h})\right)+(1-\alpha) \mathcal{R}\left(\mathbf{p}^{2}(\mathbf{h})\right) \subseteq \mathcal{R}\left(\alpha \mathbf{p}^{1}(\mathbf{h})+(1-\alpha)\left(\mathbf{p}^{2}(\mathbf{h})\right)\right)
$$

which states that any linear combination of two achievable rate tuples can be achieved by another valid power allocation policy, and is therefore in the achievable rate region, thereby 
proving the convexity.

In addition to the result given by Lemma 1, it can be shown that the rate region $\mathcal{R}$ is strictly convex, i.e., it has a curved boundary, except for the portions parallel to $R_{1}$ and $R_{2}$ axes. This result is a consequence of the following two facts. Firstly, due to the strict concavity of the logarithm, the subset relation in (20) is satisfied with strict inequality, provided $\mathbf{p}^{(1)}(\mathbf{h}) \neq \mathbf{p}^{(2)}(\mathbf{h})$ over a set of channel states with nonzero probability. Therefore, for any two corner points $\left\{R_{1}^{(1)}, R_{2}^{(1)}\right\},\left\{R_{1}^{(2)}, R_{2}^{(2)}\right\}$ on the boundary of $\mathcal{R}$, arising from distinct power control policies, there exists a rate tuple strictly outside the line connecting these two points. Therefore, if there is to be a linear portion on $\mathcal{R}$, it can only be due to a single power control policy, which in turn can only be the sum rate maximizing power control policy. Secondly, the sum rate maximizing policy yields a rectangular rate region, and therefore has only a single rate point on the boundary of $\mathcal{R}$. This fact can be proved by noting that the sum rate is maximized when the arguments of the min operation in (8) are equal, i.e., when the sum of the right hand sides of (6) and (7) is identical to the right hand side of (8) after we use the result of Proposition 1. Combining these two steps, it is easy to see that each of the points on the boundary of the rate region $\mathcal{R}$ is due to a distinct power allocation policy, and the boundary is strictly convex.

This result creates the possibility of obtaining the points on the achievable rate region boundary by maximizing a weighted sum of rates, say $R_{\boldsymbol{\mu}}=\mu_{1} R_{1}+\mu_{2} R_{2}$. The function $R_{\boldsymbol{\mu}}$ also has the same concavity properties of the sum rate, since for $\mu_{i}>\mu_{j}$, the weighted sum of rates can be written as $R_{\boldsymbol{\mu}}=\mu_{j} R_{\text {sum }}+\left(\mu_{i}-\mu_{j}\right) R_{i}$, where both $R_{\text {sum }}$ and $R_{i}$ are concave. In the next section, we obtain the optimum power control policies that achieve the points on the boundary of the achievable rate region.

\section{RATE MAXimization Via Convex Optimization}

In this section we focus on maximizing the weighted sum of rates. To illustrate both the results of the preceding section and the problem statement for this section more precisely, let us consider, without loss of generality, the case when $\mu_{1} \geq \mu_{2}$, and write down the optimization problem explicitly:

$$
\begin{aligned}
\underset{\mathbf{p}(\mathbf{h})}{\max _{1}} & \left(\mu_{1}-\mu_{2}\right)\left\{E_{1,2}\left[\log \left(1+p_{12}(\mathbf{h}) s_{12}\right)\right]\right. \\
& \left.+E_{3,4}\left[\log \left(1+p_{10}(\mathbf{h}) s_{10}\right)\right]\right\}+\mu_{2} \min \{E[\log (A)], \\
& E_{1}\left[\log \left(1+p_{12}(\mathbf{h}) s_{12}\right)+\log \left(1+p_{21}(\mathbf{h}) s_{21}\right)\right] \\
& +E_{2}\left[\log \left(1+p_{12}(\mathbf{h}) s_{12}\right)+\log \left(1+p_{20}(\mathbf{h}) s_{20}\right)\right] \\
& +E_{3}\left[\log \left(1+p_{10}(\mathbf{h}) s_{10}\right)+\log \left(1+p_{21}(\mathbf{h}) s_{21}\right)\right] \\
& \left.+E_{4}\left[\log \left(1+p_{10}(\mathbf{h}) s_{10}+p_{20}(\mathbf{h}) s_{20}\right)\right]\right\} \\
\text { s.t. } & E_{3,4}\left[p_{10}(\mathbf{h})\right]+E_{1,2}\left[p_{12}(\mathbf{h})\right]+E\left[p_{U_{1}}(\mathbf{h})\right] \leq \bar{p}_{1} \\
& E_{2,4}\left[p_{20}(\mathbf{h})\right]+E_{1,3}\left[p_{21}(\mathbf{h})\right]+E\left[p_{U_{2}}(\mathbf{h})\right] \leq \bar{p}_{2} \\
& p_{10}(\mathbf{h}), p_{12}(\mathbf{h}), p_{U_{1}}(\mathbf{h}), p_{20}(\mathbf{h}), p_{21}(\mathbf{h}), p_{U_{2}}(\mathbf{h}) \geq 0, \forall \mathbf{h}
\end{aligned}
$$

where, $E_{S}$ denotes the expectation over the event that case $S \subset\{1,2,3,4\}$ from Proposition 1 occurs, and $A$ is as given by (10). Note that the objective function is concave, and the constraint set is convex, therefore we conclude that any local optimum for the constrained optimization problem is a global optimum. Although $R_{\boldsymbol{\mu}}$ is differentiable almost everywhere since it is concave, its optimal value is attained along the discontinuity of its gradient, namely when the two arguments of the min operation are equal. This can be easily proved by contradiction: if the arguments of the minimum operation are not equal, the smaller of the arguments may be increased at the expense of the larger, just by transferring the transmit power between the cooperation signal power components $p_{U i}(\mathbf{h})$ and $p_{i j}(\mathbf{h})$ at some $\mathbf{h}$, while keeping their sum constant, and therefore not violating the average power constraints.

We get rid of the discontinuity problem of the gradient, by introducing a new variable $r$, replacing the min operation with two inequality constraints, and considering the equivalent problem

$$
\begin{aligned}
\underset{\mathbf{p}(\mathbf{h})}{\max }\left(\mu_{1}-\mu_{2}\right)\left\{E_{1,2}\left[\log \left(1+p_{12}(\mathbf{h}) s_{12}\right)\right]\right. \\
\left.+E_{3,4}\left[\log \left(1+p_{10}(\mathbf{h}) s_{10}\right)\right]\right\}+\mu_{2} r \\
\text { s.t. } r \leq E[\log (A)] \\
r \leq E_{1}\left[\log \left(1+p_{12}(\mathbf{h}) s_{12}\right)+\log \left(1+p_{21}(\mathbf{h}) s_{21}\right)\right] \\
\quad+E_{2}\left[\log \left(1+p_{12}(\mathbf{h}) s_{12}\right)+\log \left(1+p_{20}(\mathbf{h}) s_{20}\right)\right] \\
\quad+E_{3}\left[\log \left(1+p_{10}(\mathbf{h}) s_{10}\right)+\log \left(1+p_{21}(\mathbf{h}) s_{21}\right)\right] \\
\quad+E_{4}\left[\log \left(1+p_{10}(\mathbf{h}) s_{10}+p_{20}(\mathbf{h}) s_{20}\right)\right] \\
E_{3,4}\left[p_{10}(\mathbf{h})\right]+E_{1,2}\left[p_{12}(\mathbf{h})\right]+E\left[p_{U_{1}}(\mathbf{h})\right] \leq \bar{p}_{1} \\
E_{2,4}\left[p_{20}(\mathbf{h})\right]+E_{1,3}\left[p_{21}(\mathbf{h})\right]+E\left[p_{U_{2}}(\mathbf{h})\right] \leq \bar{p}_{2} \\
p_{10}(\mathbf{h}), p_{12}(\mathbf{h}), p_{U_{1}}(\mathbf{h}), p_{20}(\mathbf{h}), p_{21}(\mathbf{h}), p_{U_{2}}(\mathbf{h}) \geq 0, \forall \mathbf{h}
\end{aligned}
$$

The objective function of this new problem is concave in $\mathbf{p}(\mathbf{h})$. It can be shown that all the constraints can be written in the form $f_{i}(\mathbf{p}) \leq 0$ for convex $f_{i}(\mathbf{p})$. Therefore, the Slater's conditions for constraint qualification hold, and we can obtain the optimal solution to the constrained maximization problem (22), by using Karush-Kuhn-Tucker (KKT) conditions [19]. Although our treatment could be carried on by stating the KKT conditions for the general problem (22), in order to avoid lengthy derivations and complex expressions, without loss of generality we consider the special case of maximizing the sum rate, and illustrate the usage of KKT conditions to get some insight into the structure of the optimum solution.

\section{A. Properties of the Optimal Power Allocation: An Example}

Throughout the analysis in this subsection, we assume for simplicity of the derivations that the fading distributions are such that all realizations of the fading values satisfy $s_{12}>$ $s_{10}$ and $s_{21}>s_{20}$, i.e., the system always operates in the more interesting case 1 . This particular case is of practical interest since the cooperating transmitters are likely to be closely located with less number of scatterers and obstructions when compared to their paths to the receiver, and thus have better channel conditions among each other. We also consider maximizing the sum capacity, i.e., $\mu_{1}=\mu_{2}$. Under these 
assumptions, since $p_{10}(\mathbf{h})=p_{20}(\mathbf{h})=0$ always, the problem of maximizing the sum rate, obtained from (22) by inserting $\mu_{1}=\mu_{2}$, reduces to

$$
\begin{aligned}
\max _{\mathbf{p}(\mathbf{h})} & r \\
\text { s.t. } & r \leq E\left[\log \left(1+s_{12} p_{12}(\mathbf{h})\right)+\log \left(1+s_{21} p_{21}(\mathbf{h})\right)\right] \\
& r \leq E[\log (D)] \\
& E\left[p_{12}(\mathbf{h})+p_{U_{1}}(\mathbf{h})\right] \leq \bar{p}_{1} \\
& E\left[p_{21}(\mathbf{h})+p_{U_{2}}(\mathbf{h})\right] \leq \bar{p}_{2} \\
& p_{12}(\mathbf{h}), p_{U_{1}}(\mathbf{h}), p_{21}(\mathbf{h}), p_{U_{2}}(\mathbf{h}) \geq 0, \quad \forall \mathbf{h}
\end{aligned}
$$

where

$$
\begin{aligned}
D=1 & +s_{10}\left(p_{12}(\mathbf{h})+p_{U_{1}}(\mathbf{h})\right)+s_{20}\left(p_{21}(\mathbf{h})+p_{U_{2}}(\mathbf{h})\right) \\
& +2 \sqrt{s_{10} s_{20} p_{U_{1}}(\mathbf{h}) p_{U_{2}}(\mathbf{h})}
\end{aligned}
$$

Associating the Lagrange multipliers $\gamma_{1}, \gamma_{2}>0$ to the inequality constraints (24), (25) $\lambda_{1}, \lambda_{2}>0$ to the power constraints (26), (27), and $\xi_{i}(\mathbf{h}) \geq 0, i=1, \cdots, 4$ to the non-negativity constraints (28), and noting that the power constraints need to be satisfied by equality, we obtain the KKT conditions, which are necessary and sufficient for optimality. Note that for optimality, for any given $\mathbf{h}$, the components $p_{U_{1}}(\mathbf{h})$ and $p_{U_{2}}(\mathbf{h})$ should be either both positive or both zero. Therefore, it is sufficient to analyze these two cases: first let $p_{U_{1}}(\mathbf{h}), p_{U_{2}}(\mathbf{h})>0$. Then, the KKT conditions reduce to

$$
\begin{aligned}
& \gamma_{1} \frac{s_{12}}{1+s_{12} p_{12}(\mathbf{h})}+\gamma_{2} \frac{s_{10}}{D} \leq \lambda_{1} \\
& \gamma_{1} \frac{s_{21}}{1+s_{21} p_{21}(\mathbf{h})}+\gamma_{2} \frac{s_{20}}{D} \leq \lambda_{2} \\
& \gamma_{2} \frac{\sqrt{s_{10} s_{20} p_{U_{2}}(\mathbf{h})}+s_{10} \sqrt{p_{U_{1}}(\mathbf{h})}}{D \sqrt{p_{U_{1}}(\mathbf{h})}}=\lambda_{1} \\
& \gamma_{2} \frac{\sqrt{s_{10} s_{20} p_{U_{1}}(\mathbf{h})}+s_{20} \sqrt{p_{U_{2}}(\mathbf{h})}}{D \sqrt{p_{U_{2}}(\mathbf{h})}}=\lambda_{2}
\end{aligned}
$$

where $\gamma_{1}+\gamma_{2}=1$. From (32) and (33), it follows that there is a linear relationship between the optimal $p_{U_{1}}(\mathbf{h})$ and $p_{U_{2}}(\mathbf{h})$, i.e.,

$$
\lambda_{1}^{2} s_{20} p_{U_{1}}(\mathbf{h})=\lambda_{2}^{2} s_{10} p_{U_{2}}(\mathbf{h})
$$

Next, we include the case when $p_{U_{1}}(\mathbf{h})=p_{U_{2}}(\mathbf{h})=0$ in our analysis. The partial derivatives $\frac{\partial D}{\partial p_{U_{i}}(\mathbf{h})}, i=1,2$ are not well defined when $p_{U_{i}}(\mathbf{h})$ are both zero. However, the above derivation shows that the optimal solution is guaranteed to lie on the line given by (34), since $\left(p_{U_{1}}(\mathbf{h}), p_{U_{2}}(\mathbf{h})\right)=(0,0)$ also lies on the same line. Therefore, it is sufficient to evaluate the optimality conditions along the line (34) for $p_{U_{1}}(\mathbf{h})=$ $p_{U_{2}}(\mathbf{h})=0$, as well as for $p_{U_{1}}(\mathbf{h}), p_{U_{2}}(\mathbf{h})>0$. Substituting (32) into (30), (33) into (31), using (34), and extending (32) and (33) to include the case $p_{U_{1}}(\mathbf{h}), p_{U_{2}}(\mathbf{h})=(0,0)$, we obtain the overall set of conditions for optimality of the power allocation policy as,

$$
\begin{aligned}
& \frac{s_{12}}{1+s_{12} p_{12}(\mathbf{h})} \leq \frac{1}{\gamma_{1}} \frac{\lambda_{1}^{2} s_{20}}{\lambda_{2} s_{10}+\lambda_{1} s_{20}} \\
& \frac{s_{21}}{1+s_{21} p_{21}(\mathbf{h})} \leq \frac{1}{\gamma_{1}} \frac{\lambda_{2}^{2} s_{10}}{\lambda_{2} s_{10}+\lambda_{1} s_{20}}
\end{aligned}
$$

$$
\begin{aligned}
& \frac{1-\gamma_{1}}{D}\left(s_{10}+\frac{\lambda_{1}}{\lambda_{2}} s_{20}\right) \leq \lambda_{1} \\
& \frac{1-\gamma_{1}}{D}\left(s_{20}+\frac{\lambda_{2}}{\lambda_{1}} s_{10}\right) \leq \lambda_{2}
\end{aligned}
$$

where equalities in each of (35)-(38) hold if $p_{12}(\mathbf{h})>0$, $p_{21}(\mathbf{h})>0, p_{U_{1}}(\mathbf{h})>0, p_{U_{2}}(\mathbf{h})>0$, respectively.

These conditions shed some light onto the structure of the optimal power allocation policy: when the channel gains $s_{10}, s_{20}$ from the transmitters to the receiver are fixed, the optimal power levels associated with the components intended for the other transmitter, $p_{12}(\mathbf{h})$ and $p_{21}(\mathbf{h})$, are independent of each other and are obtained by single user waterfilling over the individual link gains $s_{12}$ and $s_{21}$, respectively. The direct link gains $s_{10}$ and $s_{20}$ determine the water level. This is easily observed by denoting the right hand sides of (35) and (36) by the constants $\nu_{1}$ and $\nu_{2}$ (since $s_{10}$ and $s_{20}$ are fixed), and solving for $p_{12}(\mathbf{h})$ and $p_{21}(\mathbf{h})$, keeping in mind the equalities are satisfied only when the power levels are positive, i.e.,

$$
\begin{aligned}
& p_{12}(\mathbf{h})=\left(\frac{1}{\nu_{1}}-\frac{1}{s_{12}}\right)^{+} \\
& p_{21}(\mathbf{h})=\left(\frac{1}{\nu_{2}}-\frac{1}{s_{21}}\right)^{+}
\end{aligned}
$$

where $(\cdot)^{+}$denotes $\max (0, \cdot)$.

Once $p_{12}(\mathbf{h})$ and $p_{21}(\mathbf{h})$ are calculated, they can be substituted into (37) and (38) with $D$ defined as in (29) to obtain $p_{U_{i}}$. On the other hand, the computation of the optimal power levels requires solving for the Lagrange multipliers $\lambda_{i}$ and $\gamma_{i}$ that satisfy the power constraints, which would require a multidimensional search. Moreover, the more general problem of maximizing the weighted sum of rates results in more complicated optimality conditions, which may be hard to solve analytically. In the next section, we obtain the optimal power allocation policy numerically using the subgradient method, and then demonstrate by simulations that the optimal power allocation policy possesses the properties described in this subsection. An interesting extension of our results could be to seek an efficient algorithm which directly obtains the Lagrange multipliers, and hence solves the KKT conditions. Another option could be solving the convex and differentiable version of the problem in (22) using the interior point methods [19].

\section{Simulation Results}

In this section we provide some numerical examples to illustrate the performance of the proposed joint power allocation and cooperation scheme. We solve the optimization problem using the method of subgradients from non-differentiable optimization theory [20], [21].

The subgradient methods are very similar to gradient ascent methods in that whenever the function is differentiable (in our case almost everywhere), the subgradient is equal to the gradient. However, their major difference from gradient ascent methods is that they are not necessarily monotonically nondecreasing. Let us denote the objective function in (21) by $R_{\mu}$. A subgradient for the concave function $R_{\boldsymbol{\mu}}(\mathbf{p})$ is any vector g that satisfies [21],

$$
R_{\boldsymbol{\mu}}\left(\mathbf{p}^{\prime}\right) \leq R_{\boldsymbol{\mu}}(\mathbf{p})+\left(\mathbf{p}^{\prime}-\mathbf{p}\right)^{\top} \mathbf{g}
$$




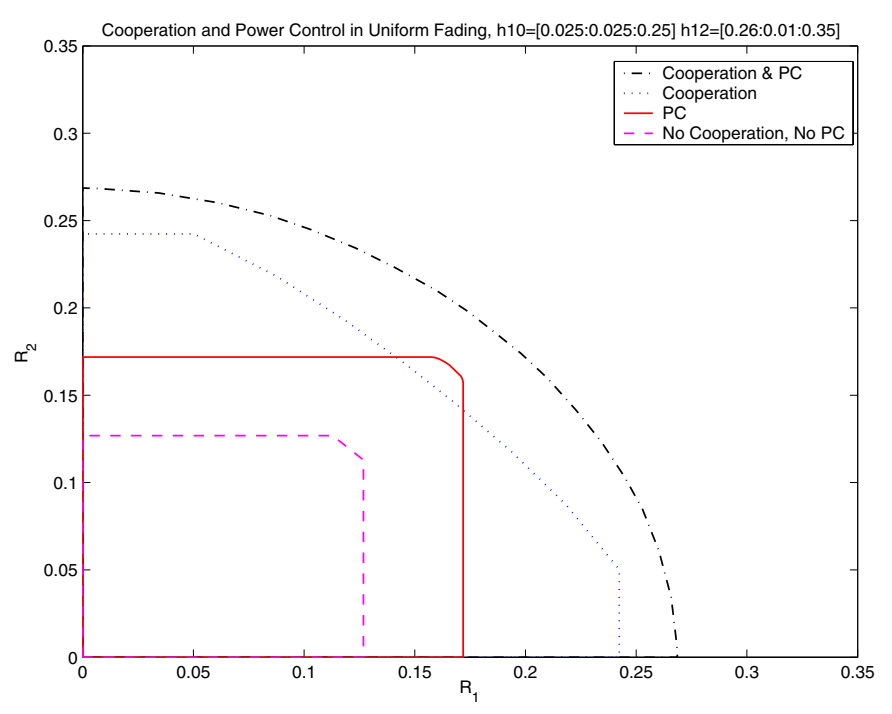

Fig. 2. Rates achievable by joint power control and user cooperation for uniform fading.

where $\mathbf{p}^{\prime}$ and $\mathbf{p}$ are two distinct arbitrary power vectors; and the subgradient method for constrained maximization uses the update

$$
\mathbf{p}(k+1)=\left[\mathbf{p}(k)+\alpha_{k} \mathbf{g}_{k}\right]^{\dagger}
$$

where $[\cdot]^{\dagger}$ denotes the Euclidean projection onto the constraint set, and $\alpha_{k}$ is the step size at iteration $k$. There are various ways to choose $\alpha_{k}$ to guarantee convergence to the global optimum; for our particular problem, we choose the diminishing stepsize, normalized by the norm of the subgradient to ensure the convergence [20]

$$
\alpha_{k}=\frac{a}{b+\sqrt{k}} \frac{1}{\left\|\mathbf{g}_{k}\right\|}
$$

Figure 2 illustrates the achievable rate region we obtain for a system with $\bar{p}_{i}=\sigma_{i}^{2}=1$, subject to uniform fading, where the links from the transmitters to the receiver are symmetric and take values from the set $\{0.025,0.050, \cdots, 0.25\}$, each with probability $1 / 10$, while the link among the transmitters is also symmetric and uniform, and takes the values $\{0.26,0.27, \cdots, 0.35\}$. Notice that here, we have intentionally chosen the fading coefficients such that the cooperation link is always better than the direct links, therefore, the system operates only in case 1 of Proposition 1. Consequently, in this particular case, our power allocation scheme is actually the optimal power allocation policy for the block Markov superposition encoding scheme.

The region for joint power control and cooperation is generated using the subgradient method with parameters $a=$ 50 and $b=5$. We carried out the optimization for various values of the priorities $\mu_{i}$ of the users, each of which give a point on the rate region boundary, and then we performed a convex hull operation over these points. We observe that power control [11]-[13] by itself improves on the rate region of the cooperative system with no power control, for rate pairs close to the sum rate, by utilizing the direct link more efficiently. Joint user cooperation and power control scheme significantly improves on all other schemes, as it takes advantage of both

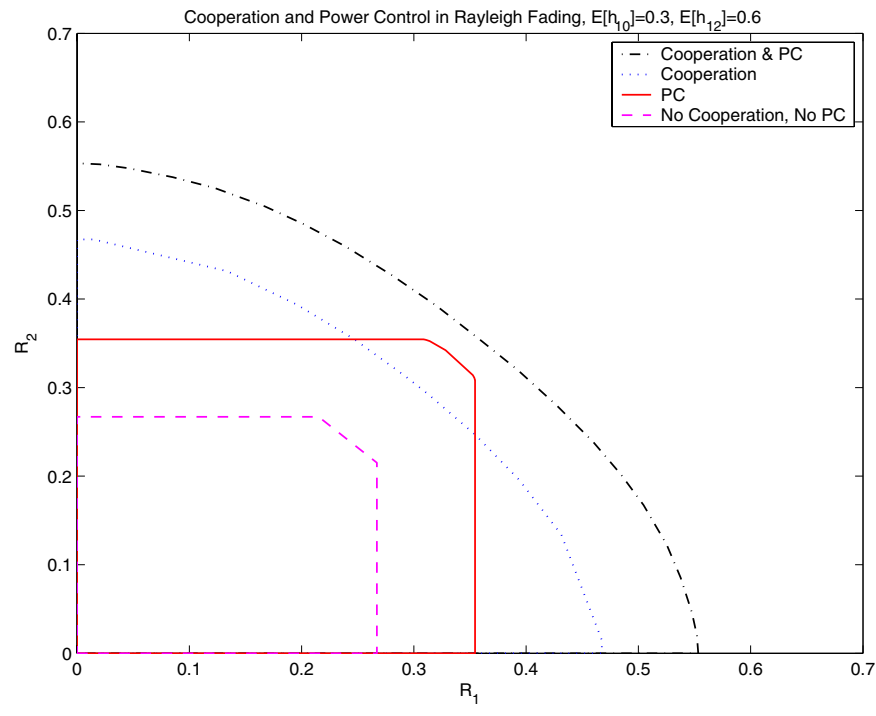

Fig. 3. Rates achievable by joint power control and user cooperation for Rayleigh fading.

cooperation diversity and time diversity in the system. In fact, we can view this joint diversity utilization as adaptively performing coding, medium accessing and routing, thereby yielding a cross-layer approach for the design of the wireless communication system.

Figure 3 also corresponds to a system with average noise and transmit power both equal to unity, but this time subject to Rayleigh fading, i.e., the power gains to the receivers are exponential random variables, with $E\left[h_{10}\right]=E\left[h_{20}\right]=0.3$, $E\left[h_{12}\right]=E\left[h_{21}\right]=0.6$. In this setting, all four cases in Proposition 1 are realized, and there is potentially some loss over the optimally achievable rates. However, we obtain a very similar set of rate regions to the uniform case, indicating that in fact the loss, if any, is very small thanks to the very low probability of both of the direct links outperforming the cooperation link.

Note that, for both of the examples in Figures 2 and 3, we have chosen relatively low signal to noise ratios. The reason for this is the well known fact that the gains from power control are especially significant at low SNR values [11]. To give an example on how much rate gain is achieved by joint use of cooperation and power control, consider the rate regions in Figure 3. It can be easily seen that when the transmitted signal and noise powers are both chosen to be equal to 1 , and the average fading value is chosen to be 0.3 , hence giving an average received SNR value of $-5.2 \mathrm{~dB}$, the rate of user 1 (or equivalently user 2) can be more than doubled when compared to a system with no power control and user cooperation. Similarly, for the same setup, the sum rate is increased from 0.48 symbols/transmission to 0.73 symbols/transmission, which is a $1.8 \mathrm{~dB}$ improvement in rate.

It is also interesting to note that in both Figures 2 and 3, cooperation with power control improves relatively less over power control only near the sum capacity. This can be attributed to the fact that, for the traditional MAC, the sum rate is achieved by time division among the users, which does not 


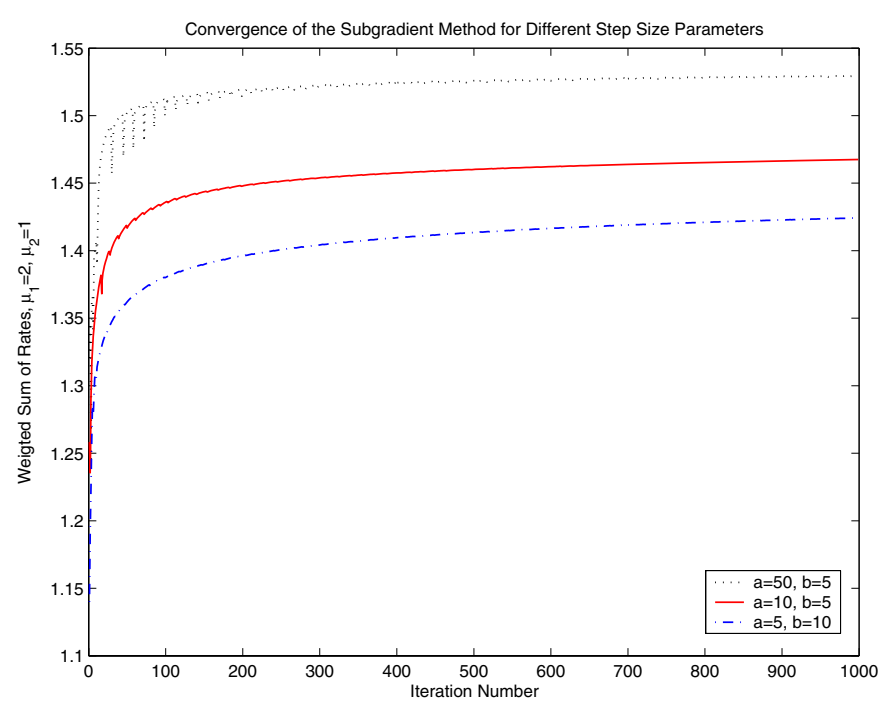

Fig. 4. Convergence of $R_{\boldsymbol{\mu}}$ using subgradient method for different step size parameters.

allow for coherent combining gain [12]. Therefore, it is not surprising to see that in order to attain cooperative diversity gain, users may have to sacrifice some of the gain they obtain from exploiting the time diversity.

In Figure 4, we illustrate the convergence of the subgradient method. The objective function is $R_{\boldsymbol{\mu}}$ with $\mu_{1}=2$ and $\mu_{2}=1$, and the step size parameters are varied. We observe that, by choosing larger step sizes, the non-monotonic behavior of the subgradient algorithm becomes more apparent, however the convergence is significantly faster than the smaller step sizes, as the algorithm is more likely to get near the optimal value of the function in the initial iterations. Note that, in our simulations we terminated the algorithm after 1000 iterations, and the three curves would eventually converge after sufficiently large number of iterations.

Figures 5(a) and 5(b) demonstrate the waterfilling nature of the optimal power levels $p_{12}(\mathbf{h})$, and $p_{21}(\mathbf{h})$, as obtained in Section 4.1. These power levels correspond to the sum rate point on Figure 2, and were obtained by using the subgradient algorithm, after 1000 iterations. The direct link gains are fixed to the values $s_{10}=0.2$ and $s_{20}=0.15$. As dictated by (35) and (36), the "water level" for $p_{12}$ is higher, and the threshold channel state level to start transmitting is lower. We see that after 1000 iterations, the subgradient approach nearly satisfies the optimality conditions.

\section{CONCLUSIONS}

We have addressed the problem of optimal power allocation for a fading cooperative MAC, where the transmitters and the receiver have channel state information, and are therefore able to adapt their coding and decoding strategies by allocating their resources. We have characterized the power control policies that maximize the rates achievable by block Markov superposition coding, and proved that, in the presence of channel state information, the coding strategy is significantly simplified: given any channel state, for each of the users, among the three signal components, i.e., those that are intended for the receiver, for the other transmitter,

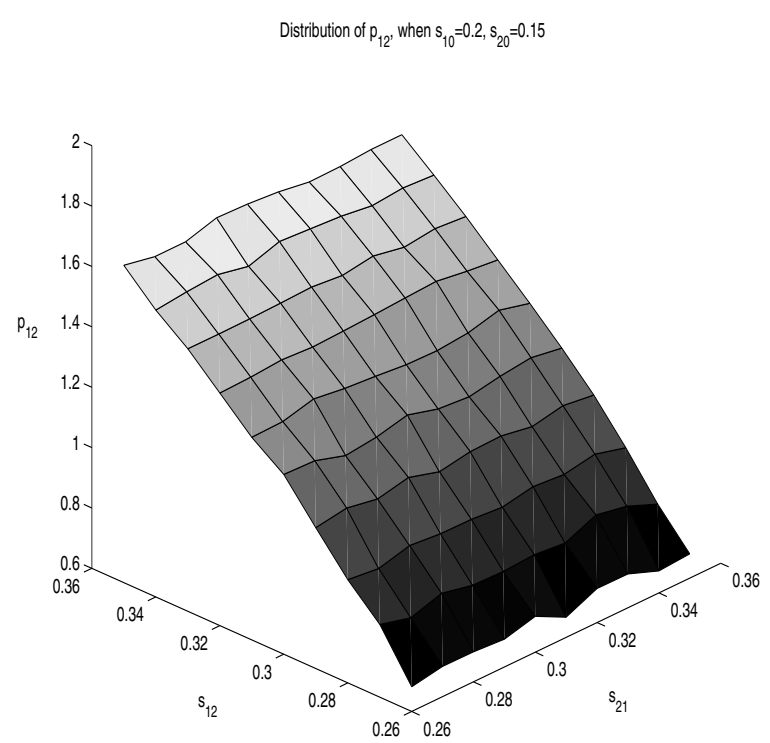

(a) The power level $p_{12}(\mathbf{h})$ as a function of $s_{12}$ and $s_{21}$.

$$
\text { Distribution of } p_{21} \text {, when } s_{10}=0.2, s_{20}=0.15
$$

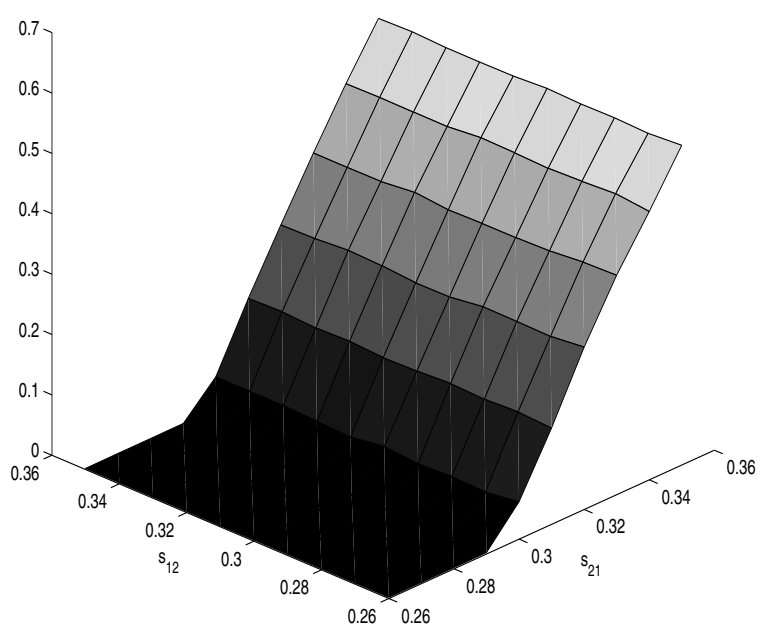

(b) The power level $p_{21}(\mathbf{h})$ as a function of $s_{12}$ and $s_{21}$.

Fig. 5. Power distributions obtained using the projected subgradient method.

and for cooperation, at least one of the first two should be allocated zero power at that channel state. This result also enabled us to formulate the otherwise non-convex problem of maximizing the achievable rates as a concave maximization (or equivalently convex minimization) problem. Using techniques from convex optimization, we obtained the analytical structure of the optimal power allocation policies. The power control policies, which are jointly optimal with block Markov coding, were then obtained through simulations. The resulting achievable rate regions for joint power control and cooperation improve significantly on cooperative systems without power control, since our joint approach makes use of both cooperative diversity and time diversity.

\section{REFERENCES}

[1] T. M. Cover and C. S. K. Leung, "An achievable rate region for the multiple access channel with feedback," IEEE Trans. Inf. Theory, vol. 27, no. 3, pp. 292-298, May 1981. 
[2] F. M. J. Willems and E. C. van der Meulen, "Partial feedback for the discrete memoryless multiple access channel," IEEE Trans. Inf. Theory, vol. 29, no. 2, pp. 287-290, March 1983.

[3] F. M. J. Willems, "The discrete memoryless multiple access channel with partially cooperating encoders," IEEE Trans. Inf. Theory, vol. 29, no. 3, pp. 441-445, May 1983.

[4] F. M. J. Willems and E. C. van der Meulen, "The discrete memoryless multiple access channel with cribbing encoders," IEEE Trans. Inf. Theory, vol. 31, no. 3, pp. 313-327, May 1985.

[5] A. B. Carleial, "Multiple access channels with different generalized feedback signals," IEEE Trans. Inf. Theory, vol. 28, no. 6, pp. 841850, Nov. 1982.

[6] F. M. J. Willems, E. C. van der Meulen, and J. P. M. Schalkwijk, "An achievable rate region for the multiple access channel with generalized feedback," in Proc. Allerton Conference, Monticello, IL, Oct. 1983.

[7] A. Sendonaris, E. Erkip, and B. Aazhang, "User cooperation diversitypart I: system description," IEEE Trans. Commun., vol. 51, no. 11, pp. 1927-1938, Nov. 2003.

[8] J. N. Laneman, D. N. C. Tse, and G. W. Wornell, "Cooperative diversity in wireless networks: efficient protocols and outage behavior," IEEE Trans. Inf. Theory, vol. 50, no. 12, pp. 3062-3080, Dec. 2004.

[9] A. Host-Madsen and J. Zhang, "Capacity bounds and power allocation for wireless relay channels," IEEE Trans. Inf. Theory, vol. 51, no. 6, pp. 2020-2040, June 2005.

[10] E. Erkip, "Capacity and power control for spatial diversity," in Proc. Conference on Information Sciences and Systems, pp. WA4-28/WA431, Princeton, NJ, March 2000.

[11] A. J. Goldsmith and P. P. Varaiya, "Capacity of fading channels with channel side information," IEEE Trans. Inf. Theory, vol. 43, no. 6, pp. 1986-1992, Nov. 1997.

[12] R. Knopp and P. A. Humblet, "Information capacity and power control in single-cell multiuser communications," in Proc. IEEE ICC, June 1995

[13] S. Hanly and D. N. C. Tse, "Multiaccess fading channels-part I: polymatroid structure, optimal resource allocation and throughput capacities," IEEE Trans. Inf. Theory, vol. 44, no. 7, pp. 2796-2815, Nov. 1998.

[14] E. Biglieri, J. Proakis, and S. Shamai (Shitz), "Fading channels: information-theoretic and communications aspects," IEEE Trans. Inf. Theory, vol. 44, no. 6, pp. 2619-2692, Oct. 1998.

[15] L. L. Xie and P. R. Kumar, "A network information theory for wireless communication: scaling laws and optimal operation," IEEE Trans. Inf. Theory, vol. 50, no. 5, pp. 748-767, May 2004.

[16] L. Sankaranarayanan, G. Kramer, and N. B. Mandayam, "Capacity theorems for the multiple-access relay channel," in Proc. 42nd Annual Allerton Conference on Communication, Control, and Computing, Montocello, IL, Sept. 29-Oct 1, 2004.
[17] O. Kaya, "Window and backwards decoding achieve the same sum rate for the fading cooperative Gaussian multiple access channel," in Proc. IEEE Global Communications Conference, San Francisco, CA, Nov. 2006

[18] J. N. Laneman and G. Kramer, "Window decoding for the multiple access channel with generalized feedback," in Proc. IEEE ISIT 2004, pp. 279, Chicago, June 27-July 2, 2004.

[19] S. Boyd and L. Vandenberghe, Convex Optimization. Cambridge University Press, 2004.

[20] N. Z. Shor, Minimization Methods for Non-Differentiable Functions. Springer-Verlag, 1979.

[21] D. P. Bertsekas, Nonlinear Programming. Athena Scientific, 1995.

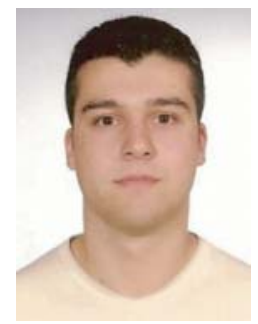

cations.

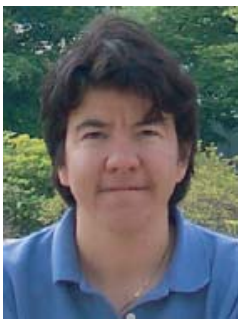

Onur Kaya (S'02, M'06) received the B.S. degree in electrical and electronics engineering from Bilkent University, Ankara, Turkey, in 2000, and the M.S. and Ph.D. degrees in electrical and computer engineering from University of Maryland, College Park, in 2002 and 2005 respectively.

Currently he is with the Electronics Engineering Department at Işık University in Istanbul, Turkey as an Assistant Professor. His research interests are in the fields of communication theory and information theory, with particular focus on wireless communi-

Sennur Ulukus (S'90, M'98) received the B.S. and M.S. degrees in electrical and electronics engineering from Bilkent University, Ankara, Turkey, in 1991 and 1993, respectively, and the Ph.D. degree in electrical and computer engineering from Rutgers University, NJ, in 1998. During her Ph.D. studies, she was with the Wireless Information Network Laboratory (WINLAB), Rutgers University. From 1998 to 2001, she was a Senior Technical Staff Member at AT\&T Labs-Research in NJ. In 2001, she joined the University of Maryland at College Park, where she is currently an Associate Professor in the Department of Electrical and Computer Engineering, with a joint appointment at the Institute for Systems Research (ISR). Her research interests are in wireless communication theory and networking, network information theory for wireless networks, signal processing for wireless communications, and security for multi-user wireless communications.

Sennur Ulukus is a recipient of the 2005 NSF CAREER Award, and a co-recipient of the 2003 IEEE Marconi Prize Paper Award in Wireless Communications. She serves as an Associate Editor for the IEEE Transactions on Communications since 2003, as a Guest Editor for the IEEE Journal on Selected Areas in Communications in 2007, as the co-chair of the Communication Theory Symposium at the 2007 IEEE Global Telecommunications Conference and as the Secretary of the IEEE Communication Theory Technical Committee (CTTC). 